\title{
Quantum nondemolition photon detection in circuit QED and the quantum Zeno effect
}

\author{
Ferdinand Helmer, ${ }^{1}$ Matteo Mariantoni, ${ }^{2,3}$ Enrique Solano, ${ }^{1,4}$ and Florian Marquardt ${ }^{1}$ \\ ${ }^{1}$ Department of Physics, Center for NanoScience, and Arnold Sommerfeld Center, Ludwig-Maximilians-Universität, \\ Theresienstrasse 37, D-80333 Munich, Germany \\ ${ }^{2}$ Walther-Meissner-Institut, Bayerische Akademie der Wissenschaften, Walther-Meissner-Str. 8, D-85748 Garching, Germany \\ ${ }^{3}$ Physics Department, Technische Universität München, James-Franck-Str., D-85748 Garching, Germany \\ ${ }^{4}$ Departamento de Quimica Fisica, Universidad del Pais Vasco-Euskal Herriko Unibertsitatea, 48080 Bilbao, Spain
}

(Received 17 December 2007; revised manuscript received 15 January 2009; published 20 May 2009)

\begin{abstract}
We analyze the detection of itinerant photons using a quantum nondemolition measurement. An important example is the dispersive detection of microwave photons in circuit quantum electrodynamics, which can be realized via the nonlinear interaction between photons inside a superconducting transmission line resonator. We show that the back action due to the continuous measurement imposes a limit on the detector efficiency in such a scheme. We illustrate this using a setup where signal photons have to enter a cavity in order to be detected dispersively. In this approach, the measurement signal is the phase shift imparted to an intense beam passing through a second cavity mode. The restrictions on the fidelity are a consequence of the quantum Zeno effect, and we discuss both analytical results and quantum trajectory simulations of the measurement process.
\end{abstract}

DOI: 10.1103/PhysRevA.79.052115

PACS number(s): 03.65.Ta, 03.65.Xp, 42.50.Lc

\section{INTRODUCTION}

Quantum nondemolition (QND) measurements are ideal projective measurements that reproduce their outcome when repeated $[1,2]$. Using them, it is possible to measure the state of a system with the minimal disruption required by quantum mechanics. Recent successful experimental demonstrations of QND detection for superconducting qubits and microwave photons [3-6] are both of fundamental interest and crucial for the development of quantum communication and information processing. When QND detection is applied continuously to a system that would otherwise undergo some intrinsic dynamics, quantum jumps are observed, tracing the quantum evolution in real time [7-11]. As a consequence, the dynamics tends to be frozen, a result now known as the quantum Zeno effect [12-17].

In the present paper, we show that the interplay of these phenomena may put interesting constraints on the detection of itinerant quanta. The specific minimal example we will discuss concerns the continuous dispersive QND detection of single photons passing through a cavity. The crucial distinction to be recognized is the following. For localized quanta (e.g., a photon already created inside a cavity $[8,18]$ ), the quantum Zeno effect could presumably only enhance the detection by suppressing the decay. However, this no longer holds for the detection of itinerant quanta if we require that our detector is always working and can detect the quantum without knowing the arrival time in advance. As we will show, in this case the unavoidable back action of the measurement device produces a quantum Zeno effect, suppressing the fidelity of measurements.

Similar measurement physics is involved when trying to read out the phonon state of a nanoresonator using a single electron transistor (SET) and a Cooper pair box (CPB) as a coupler [19]. However, there are some distinct differences to the scheme considered here. The most prominent difference is that we analyze a scheme to detect itinerant quanta (in our case photons) and therefore do not assume the signal mode to be already prepared in a certain state. Although the quantum Zeno effect also plays a role in the CPB dynamics in [19], the conclusions and reasoning for the optimal measurement rate in our case are different.

Such dispersive photon detection schemes could have a particularly important application in the field of quantum electrodynamics (QED) in superconducting circuits. Circuit QED setups [12,20-22] offer the possibility to implement ideas of quantum optics using microwave photons propagating in transmission lines and they also could form the basis for novel architectures capable of scalable, fault-tolerant quantum computing [23]. Proposals for generating nonclassical photon states exist or have been implemented $[4,21,24,25]$. However, the on-chip single-shot detection of itinerant photons is still missing.

Recent experiments have demonstrated dispersive qubit detection [3] and measurements of photon statistics [4]. Based on the dispersive coupling strengths demonstrated there, one could employ a superconducting qubit inside a microwave transmission line resonator to induce a nonlinear coupling between two modes of the resonator (or alternatively couple two cavities), thus creating a dispersive photon detector of the type discussed here. Even though we find a limit for the photon detection fidelity of about 30\% for the simplest scheme considered here, this would still be a considerable improvement over the present state of the art.

\section{MODEL}

We investigate a QND scheme utilizing the nonlinear Kerr-type coupling [26-28] of two discrete localized modes of a bosonic field. The presence of a quantum inside the signal mode gives rise to a frequency shift of the detection mode, which can be observed dispersively via the phase shift of a beam transmitted through that mode (see Fig. 1). In turn, the signal mode frequency fluctuates due to the detection beam's shot noise. As a consequence, the incoming signal photon will be reflected with a probability that rises with 


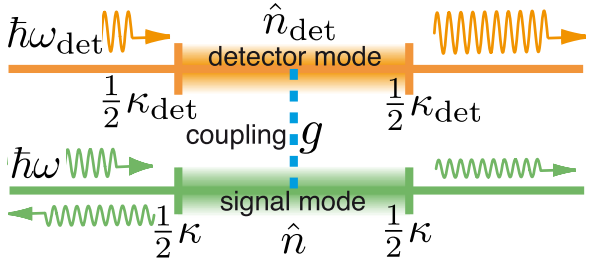

FIG. 1. (Color online) Schematic sketch of the model. Two cavity modes are coupled anharmonically. The detector mode is irradiated with a strong coherent field that suffers a phase shift whenever a photon is present in the signal mode.

coupling strength and detection beam intensity.

This incarnation of the quantum Zeno effect generates a trade-off that yields the highest detection efficiency at intermediate coupling strengths. In that way, such dispersive schemes for itinerant quanta turn out to be similar to weak measurements using general linear detectors and amplifiers [2].

We proceed as follows. (i) We numerically evaluate quantum jump trajectories for the phase-shift signal in a minimal model of a QND photon detector and analyze the fraction of detected photons, observing the trade-off described above. (ii) We interpret these findings using an analytical approximation. (iii) Finally, we briefly comment on possible experimental realizations.

We consider a system of two cavity modes with a Kerrtype coupling of strength $g$,

$$
\hat{H}=\hbar \omega\left(\hat{n}+\frac{1}{2}\right)+\hbar \omega_{\mathrm{det}}\left(\hat{n}_{\mathrm{det}}+\frac{1}{2}\right)+\hbar g \hat{n} \hat{n}_{\mathrm{det}}+\hat{H}_{\mathrm{drive}+\mathrm{dec} a y}
$$

These modes might represent two different electromagnetic field modes inside an optical or microwave cavity, the modes of two adjacent cavities [29], or even two anharmonically coupled modes of a nanomechanical resonator. Photons in the signal mode (frequency $\omega$, number operator $\hat{n}$ ) and the detector mode $\left(\omega_{\text {det }}, \hat{n}_{\text {det }}\right)$ decay by leaking out of the cavity. The anharmonic Kerr-type coupling arises generically when introducing any nonlinear medium, such as an atom, a qubit, or a quantum dot, into a cavity and has been studied for the purpose of QND measurements in quantum optics [26-28]. It induces a phase shift in the strong detection beam $\left(\left\langle\hat{n}_{\text {det }}\right\rangle\right.$ $\gg 1$ ) upon presence of a signal photon.

We are interested in analyzing individual realizations of the phase-shift signal as a function of time. The phase shift can be observed by continuously measuring an appropriate field quadrature of the detection beam (e.g., in a homodyne setup). As the beam passes through the cavity, the beam becomes entangled weakly with the cavity's state. Thus, the stochastic measurement outcomes reveal information about that state, feeding back into the time evolution of the cavity's density matrix. This physics is described by a stochastic master equation $[2,18,30-32]$ for the density matrix $\hat{\rho}$ conditioned on the output signal (see [18]).

\section{STOCHASTIC MASTER EQUATION}

In this section we derive a stochastic master equation for this measurement situation. Starting from a standard Lindblad master equation, involving both driving and decay for the two modes, we can derive a stochastic master equation that keeps track of the individual measurement signal obtained in each individual run of the experiment. Before presenting that equation [Eq. (2) below], let us briefly describe in qualitative terms the purpose of using a stochastic master equation. Imagine a general measurement situation where the system in question cannot be directly projectively measured, but instead one has to couple a measurement apparatus (most often a meso- or macroscopic apparatus) to the system. Only this apparatus can then be projectively measured. Assuming that apparatus and coupling to the system are indeed suitable to extract information about a certain system observable via a projective measurement on an apparatus observable, during such a measurement the following happens: We let system and apparatus, initially assumed to be in a product state, interact according to the coupling Hamiltonian for some time interval $\Delta t$. Afterwards, the state will have changed, generally into an entangled state between system and measurement apparatus. It is by this entanglement that information about the system state is transferred into the measurement apparatus. Then we imagine to projectively measure the measurement apparatus. This has several consequences: (i) the measurement apparatus experiences a projection onto one of the (usually smoothly distributed) eigenstates of the apparatus observable; (ii) the system and the measurement apparatus are now again in a product state; (iii) this evidently also changed the system state (this is called measurement back action), however not necessarily into an eigenstate of the system. It is important to realize that this change is also random, as it is conditional on the measurement result we obtained when projectively measuring the apparatus.

Very often and especially in the situation we consider, the coupling between system and measurement apparatus is weak, meaning that during one measurement as we just described, the system state only changes very little. This is then referred to as a "weak" measurement. When we now imagine that we take the continuum limit $\Delta t \rightarrow 0$, it is intuitively clear that the infinitesimally small kicks the system experiences should lead to stochastic dynamics. We can therefore expect (i) having a stochastic term enter the master equation which leads to (ii) diffusionlike behavior of the system state and (iii) eventually the system will be driven into an eigenstate of the system observable we indirectly measured. The time scale on which this indirect projection happens corresponds exactly to the time scale on which we could extract enough information out of the measurement signal (the sequence of results from projectively measuring the apparatus) to infer the value of the system observable with certainty. On this time scale, we have indirectly performed a QND measurement of the system. No matter how much longer we keep on measuring, the result of subsequent measurements will always give the same result, as the system has been projected.

In addition to the back action modifying the quantum dynamics, we also obtain the classical measurement signal. It is again intuitively clear that this signal should be noisy (it is a 
sequence of random projections of the measurement apparatus) but should contain the same realization of the noise as the internal back-action dynamics.

To summarize, this method of analyzing weak measurements gives us access to concrete realizations of the classical noisy signal while we also obtain the system internal measurement back-action modified quantum dynamics for each of those realizations. This allows to design and validate experimentally realistic detection schemes in a powerful way and is the method of choice for the present analysis.

In this derivation we follow [18,33-35], adapted to the case of zero temperature and a two-sided cavity. As a starting point we write down a master equation to model the quantum dynamics of the system introduced in Fig. 1 and Eq. (1),

$$
\begin{aligned}
\dot{\hat{\rho}}= & -i\left[\frac{\kappa_{1}}{2}\left(\alpha \hat{a}_{1}^{\dagger}+\alpha^{*} \hat{a}_{1}\right)+g \hat{a}_{1}^{\dagger} \hat{a}_{1} \hat{a}_{0}^{\dagger} \hat{a}_{0}, \hat{\rho}\right] \\
& +\kappa\left(\hat{a}_{0} \hat{\rho} \hat{a}_{0}^{\dagger}-\frac{1}{2} \hat{a}_{0}^{\dagger} \hat{a}_{0} \hat{\rho}-\frac{1}{2} \hat{\rho} \hat{a}_{0}^{\dagger} \hat{a}_{0}\right) \\
& +\kappa_{1}\left(\hat{a}_{1} \hat{\rho} \hat{a}_{1}^{\dagger}-\frac{1}{2} \hat{a}_{1}^{\dagger} \hat{a}_{1} \hat{\rho}-\frac{1}{2} \hat{\rho} \hat{a}_{1}^{\dagger} \hat{a}_{1}\right) \\
& -i \sqrt{\dot{N}_{\mathrm{in}} / 2 \kappa}\left[\hat{a}_{0}+\hat{a}_{0}^{\dagger}, \hat{\rho}\right] .
\end{aligned}
$$

The first Hamiltonian term describes driving of the detector mode (annihilation operator $\hat{a}_{1}$, decay rate $\kappa_{1}$ ) as well as the Kerr-type nonlinear coupling between the modes. The driving amplitude $\alpha$ results in a coherent state in the signal mode with an average photon number $|\alpha|^{2}$. The signal mode annihilation operator is denoted as $\hat{a}_{0}$ and the its decay rate is labeled $\kappa$. The second and third terms are the Lindblad-decay terms for both modes reflecting the finite $Q$ factor of the cavity. The fourth term is a weak driving of the signal mode to model the itinerant photons impinging onto the cavity. Here, $\dot{N}_{\text {in }}$ describes the rate of itinerant photons impinging onto the cavity from the outside. Although the drive is coherent, after a suitable unraveling of the master equation it will become apparent that the Poissonian nature of the coherent drive will indeed model the situation we have in mind correctly-namely, that once in a while a photon comes along the transmission line, hits the cavity, and can then be detected or not. Note that we have chosen to work in an interaction picture and in a rotating frame to eliminate the explicit time dependence of the drives.

It is convenient to follow the steps of [18,33-36] to (i) derive the unraveled version of Eq. (A1) and to (ii) adiabatically eliminate the detector mode from this equation assuming $\kappa_{\text {det }} \gg \kappa$. This is done in the Appendix. Finally, we obtain a stochastic master equation for the signal mode alone, greatly facilitating the numerical study of the detection efficiency. Its stochastic term accounts for the back action of the measurements performed on the field quadrature leaking out of the detector mode correctly and reads

$$
\begin{aligned}
\dot{\hat{\rho}}_{s}= & -i \sqrt{\frac{\dot{N}_{\text {in }} \kappa}{2}}\left[\hat{a}+\hat{a}^{\dagger}, \hat{\rho}\right]+\kappa\left(\hat{a} \hat{\rho} \hat{a}^{\dagger}-\frac{1}{2} \hat{n} \hat{\rho}-\frac{1}{2} \hat{\rho} \hat{n}\right) \\
& -2 \Gamma[\hat{n},[\hat{n}, \hat{\rho}]]-\sqrt{4 \Gamma}[\hat{n} \hat{\rho}+\hat{\rho} \hat{n}-2 \hat{\rho}\langle\hat{n}\rangle(t)] \xi(t),
\end{aligned}
$$

while the classical noisy measurement signal that corresponds to this internal dynamics is given by

$$
X(t) \equiv\langle\hat{n}\rangle(t)+\frac{1}{4} \sqrt{\frac{1}{\Gamma}} \xi(t) .
$$

\section{ANALYSIS OF DETECTION EFFICIENCY}

We analyze a situation with a continuous weak coherent beam of photons entering at a rate $\dot{N}_{\text {in }}$ into the signal mode, whose intensity decay rate is $\kappa$ [first line of Eq. (3)]. We have chosen to work in the limit of a large detector mode decay rate, $\kappa_{\text {det }} \gg \kappa$, which is favorable for the detection process and makes it possible to adiabatically eliminate that mode [18], keeping only the signal mode $\hat{n}=\hat{a}^{\dagger} \hat{a}$ and drastically reducing the numerical effort. After adiabatic elimination, the coupling strength $g$ and the detection beam intensity are combined into the measurement rate [18]

$$
\Gamma \equiv g^{2}\left\langle\hat{n}_{\mathrm{det}}\right\rangle /\left(4 \kappa_{\mathrm{det}}\right),
$$

where $1 / \Gamma$ is the time scale needed to resolve different photon numbers. The last, stochastic term in Eq. (3), describes the measurement back action.

It contains a systematic term depending on the average number of signal photons, as well as a stochastic term representing the unavoidable vacuum noise, where $\langle\xi\rangle=0$ and $\left\langle\xi(t) \xi\left(t^{\prime}\right)\right\rangle=\delta\left(t-t^{\prime}\right)$. In deriving Eqs. (3) and (4), we have assumed that the transmitted and reflected signals are superimposed symmetrically to extract the maximum information.

As in any measurement of field quadratures, temporal filtering is required to suppress the noise. We average over a time span $\tau_{\text {avg }}$, which should be as large as possible while still remaining smaller than the expected temporal extent of the phase-shift signal due to a single photon, i.e., $\tau_{\text {avg }}$ not much bigger than $\kappa^{-1}$. We denote the averaged signal as $\bar{X}(t)$.

\section{A. Numerical results}

We numerically solve the master equation, using it to compute the signal $\bar{X}(t)$ and the occupation of the signal mode $\langle\hat{n}\rangle(t)$ as a function of time. We then implement the minimal model of a threshold detector. Time points when the quantum jump trajectory $\bar{X}(t)$ first exceeds the threshold $X_{\text {thr }}$ are counted as detection events, and the detector is then set insensitive for a dark time $\tau_{\text {dark }}$, suitably chosen to avoid multiple detection, i.e., $\dot{N}_{\text {in }}^{-1} \gg \tau_{\text {dark }} \gg \kappa^{-1}$.

Our discussion will focus on small values of $\dot{N}_{\text {in }}$, making the results independent of $\tau_{\text {dark, }}$, while we will analyze the dependence on $X_{\text {thr }}$ in some details. In Fig. 2 we show two example trajectories. Whereas the expected number of signal photons is the same for both cases, the increase in the measurement rate $\Gamma / \kappa$ decreases the number of photons actually detected, while at the same time enhancing the signal-tonoise ratio in the trajectory. This is an indication of the quantum Zeno effect in the detection of itinerant quanta, which we now want to study in more quantitative details.

We plot the rate of photon detection events $\dot{N}_{\text {det }}$ versus the rate of incoming photons $\dot{N}_{\text {in }}$ [Fig. 3(a)]. The detection efficiency $\eta$ is naturally defined as the ratio of detected vs in- 


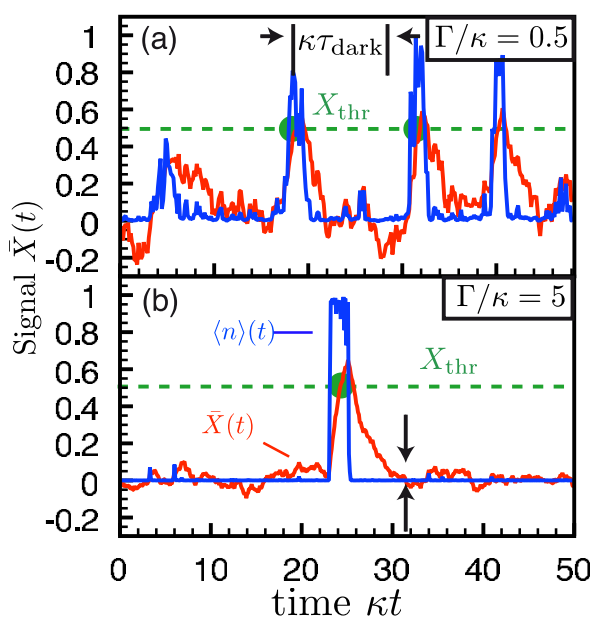

FIG. 2. (Color online) Quantum jump trajectories illustrating dispersive photon detection. The observable homodyne signal $\bar{X}(t)$ [red (light gray) lines] and the corresponding signal mode occupation $\langle\hat{n}\rangle(t)$ [blue (dark gray) lines], for two different values of the measurement rate $\Gamma / \kappa$ at a fixed input rate $\dot{N}_{\text {in }}$. Photon detection events are indicated as filled circles. The relative noise strength $\propto\left(\Gamma \tau_{\text {avg }}\right)^{-1 / 2}$ is suppressed with increasing $\Gamma / \kappa$, but the number of photons actually detected also decreases due to the quantum Zeno effect (see main text). The size of the noise floor, the detector threshold $X_{\text {thr }}$ [(green) dashed line], and the dark time are indicated. Here and in the following plots $\kappa \tau_{\text {avg }}=2$.

coming photons, obtained at small input rates $\dot{N}_{\text {in }}$,

$$
\left.\eta \equiv \frac{d \dot{N}_{\text {det }}}{d \dot{N}_{\text {in }}}\right|_{\dot{\mathrm{N}}_{\mathrm{in}}=0} .
$$

Figure 3(c) displays the efficiency $\eta$ as a function of $\Gamma / \kappa$ and $X_{\mathrm{thr}}$. The statistics for this figure were obtained from extensive numerical simulations by generating $O\left(10^{4}\right)$ trajectories of length $10^{2} / \kappa$ for seven different rates $\dot{N}_{\text {in }}$ at each value of $\Gamma / \kappa$. Apparently, the detector efficiency $\eta$ is strongly suppressed both for $\Gamma / \kappa \ll 1$ (low signal-to-noise ratio) and $\Gamma / \kappa \gg 1$.

\section{B. Analytical results}

To interpret these results, we now calculate the total transmission probability through the signal mode, whose frequency fluctuates due to the shot noise in the detection mode, which is treated as classical noise. We start from the semiclassical equation of motion for the complex field amplitude $\alpha(t)$ in the signal mode,

$$
\dot{\alpha}(t)=\left(-i \delta \omega(t)-\frac{\kappa}{2}\right) \alpha(t)+\sqrt{\frac{\kappa}{2}} \alpha_{L}^{\text {in }} .
$$

Here $\alpha_{L}^{\text {in }}$ is the amplitude of the signal photon field entering the cavity from the left side and $\delta \omega(t) \equiv g n_{\text {det }}(t)$ is the fluctuating frequency shift $\left(n_{\operatorname{det}} \gg 1\right)$. The correlator of the noise is given by
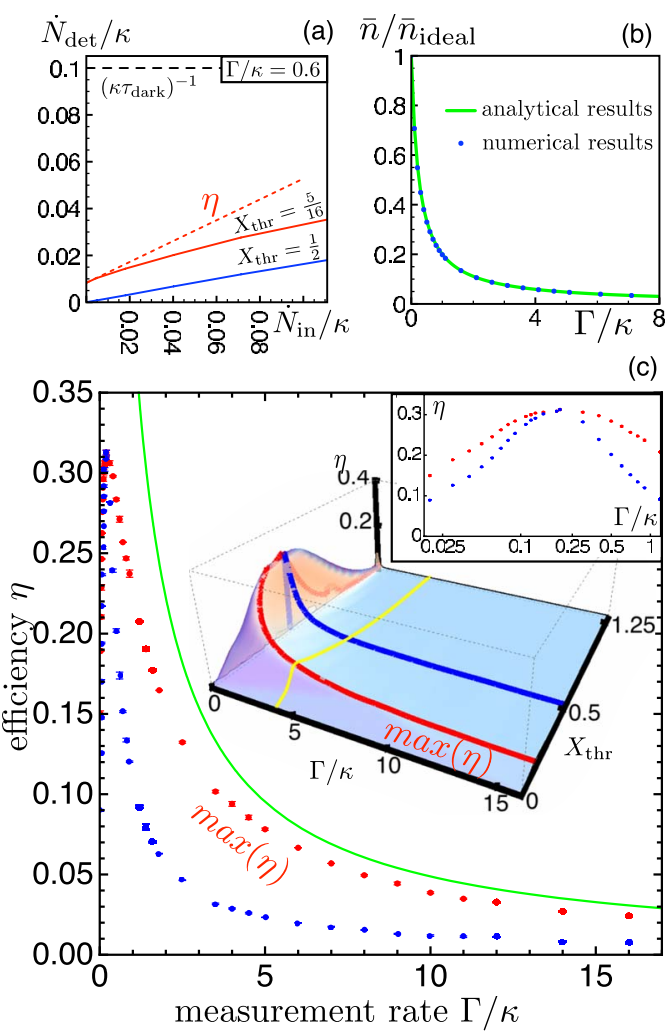

FIG. 3. (Color online) (a) Detector profile: Rate of detected vs incoming photons, at $\Gamma / \kappa=0.6$, for two different thresholds $X_{\mathrm{thr}}$. Observe the dark count rate (offset at $\dot{N}_{\text {in }}=0$ ), the detector efficiency $\eta$ defined from the slope at $\dot{N}_{\text {in }}=0$, and the saturation for large $\dot{N}_{\text {in }} \sim \tau_{\text {dark }}^{-1}$. (b) Suppression of the signal photon number $\bar{n}$ inside the cavity as a function of measurement rate $\Gamma / \kappa$ compared to perfect transmission $\left(\bar{n}_{\text {ideal }}\right)$. (c) Detector efficiency $\eta$, obtained from quantum trajectory simulations, as a function of $\Gamma$ and $X_{\text {thr }}$ (3D inset), and comparison to the analytical results (main plot). The blue (lower line of) data points display $\eta$ for fixed $X_{\text {thr }}=0.5\left(X_{\text {thr }}\right.$ $=0.5$ cut in $3 \mathrm{D}$ inset). When maximizing the $\eta$ over $X_{\text {thr }}$ for any given $\Gamma / \kappa$, the red (upper line of) data points are obtained [labeled " $\max (\eta) "]$, agreeing well with the analytical asymptote [green (solid) thin line] at higher values of $\Gamma / \kappa$. Small inset: Lin-log enlargement of the region of the maximum efficiency of the main plot. (Here, the $x$ axis is scaled logarithmically while the $y$ axis is scaled linearly to obtain the optimum resolution of the peak structure.)

$$
\langle\delta \omega(t) \delta \omega(0)\rangle-\langle\delta \omega\rangle^{2}=g^{2} \bar{n}_{\operatorname{det}} e^{-\kappa_{\operatorname{det}}|t| / 2} .
$$

To obtain an expression for the transmission probability, we write down the formal solution for $\alpha(t)$,

$$
\frac{\alpha(t)}{\sqrt{\kappa_{L}} \alpha_{L}^{\text {in }}}=\int_{-\infty}^{t} d t^{\prime} \exp \left[-i \int_{t^{\prime}}^{t} \delta \omega\left(t^{\prime \prime}\right) d t^{\prime \prime}-\frac{\kappa}{2}\left(t-t^{\prime}\right)\right] .
$$

Note that the fluctuations $\delta \omega(t)$ themselves are nonGaussian. Still, the integral in the exponent is approximately Gaussian for time intervals that fulfill $\kappa_{\text {det }}\left|t-t^{\prime}\right| \gg 1$ due to the central limit theorem. These times yield the main contribution under our assumption of a "fast detector," $\kappa_{\text {det }} \gg \kappa$. Thus, we can evaluate $\left\langle|\alpha|^{2}\right\rangle$ using the formula $\langle\exp [-i Y]\rangle$ $=\exp \left[-i\langle Y\rangle-\frac{1}{2} \operatorname{Var} Y\right]$ for a Gaussian random variable $Y$ and 
inserting Eq. (8). From this, we obtain the average transmitted intensity

$$
\left\langle\left|a_{R}^{\text {out }}\right|^{2}\right\rangle=\frac{\kappa}{2}\left\langle|\alpha|^{2}\right\rangle=\langle\mathcal{T}\rangle\left|\alpha_{L}^{\text {in }}\right|^{2}
$$

and the average transmission probability

$$
\langle\mathcal{T}\rangle=\left(1+4 \frac{\Gamma}{\kappa}\right)^{-1} .
$$

Before we can correlate the suppression of the transmission with the reduction of the detector efficiency $\eta$ in the limit of $\Gamma / \kappa \gg 1$, one more consideration is necessary. In this limit, any photon that has entered the cavity will almost certainly be detected. Once detected, the photon loses the coherence with the incoming beam, which is needed for perfect transmission on resonance in the ideal coherent case. As a consequence, it acquires an equal probability to leave the cavity through the left or the right port. This means that, on average, the number of detected photons is twice the number of transmitted photons. The expected relation is thus $\eta=2\langle\mathcal{T}\rangle$, which is indeed observed nicely when comparing to the numerical data [Fig. 3(c)].

The reduction of detector efficiency at $\Gamma / \kappa \gg 1$ thus has found its explanation in the quantum Zeno effect: many photons remain undetected because they are reflected due to detector back action. As low values of $\Gamma / \kappa$ are also unfavorable, due to a bad signal-to-noise ratio, the maximum efficiency is found near the intermediate value $\Gamma / \kappa=1 / 4$ (see Fig. 3).

\section{Possible realization in superconducting circuit quantum electrodynamics setups}

Cavity QED setups in superconducting circuits $[12,20-22]$ have been used to implement ideas of quantum optics on the chip and are considered a promising candidate for scalable, fault-tolerant quantum computing [23]. While proposals for generating nonclassical photon states exist or have been implemented $[4,21,24,25]$, the on-chip single-shot detection of itinerant photons is still missing.

Building on recent experiments that demonstrated dispersive qubit detection [3] and measurements of photon statistics [4], one could employ the superconducting qubit to induce a nonlinear coupling between two modes of the microwave transmission line resonator (or coupling two cavities [37]), thus creating a dispersive photon detector of the type discussed here.

These experiments realize a Jaynes-Cummings coupling between qubit and resonator of up to $2 \pi \times 100 \mathrm{MHz}$, resonators with frequencies of about $2 \pi \times 5 \mathrm{GHz}$, and a large spread of resonator decay rates $\kappa$ between 1 and $100 \mathrm{MHz}$. To make the example more concrete we suggest to employ the qubit as a nonlinear coupler between two modes of a transmission line cavity.

Imagine the qubit being tuned into resonance with the signal mode, such that their resonances hybridize, as described by the Jaynes-Cummings model. The detector mode is assumed to be far detuned from the qubit and the signal mode, i.e., we consider the case of dispersive coupling. The unperturbed Hamiltonian $\hat{H}_{0}$ [redefined to absorb the vacuum energy of the harmonic oscillators and using rotating wave approximation (RWA)] thus reads

$$
\hat{H}_{0} \equiv \hbar \frac{\omega_{q}}{2} \hat{\sigma}_{z}+\hbar \omega_{\alpha} \hat{a}_{\alpha}^{\dagger} \hat{a}_{\alpha}+\hbar \omega_{\beta} \hat{a}_{\beta}^{\dagger} \hat{a}_{\beta}+g_{0, \alpha}\left(\hat{\sigma}^{+} \hat{a}_{\alpha}+\hat{a}_{\alpha}^{\dagger} \hat{\sigma}^{-}\right)
$$

This contains the resonant coupling of the qubit to the signal mode (mode index $\alpha$ ), while the coupling to the detection mode (index $\beta$ ) will be considered perturbatively. Now imagine the incoming signal photon being on resonance with one of the states of the coupled system (qubit/signal mode), e.g., $\left|\alpha^{-}\right\rangle \equiv 1 / \sqrt{2}(|0, \uparrow\rangle+|1, \downarrow\rangle) \otimes\left|n_{\beta}\right\rangle$. This state is an eigenstate of the qubit-signal mode system which is only weakly (dispersively) coupled to the detector mode. Provided we consider only situations with at most one signal photon inside the signal mode $(\alpha)$ and $n_{\beta}$ photons in the detection $(\beta)$ mode (as was the case in the preceding discussion), we can restrict ourselves to the subspace $\{|0, \downarrow\rangle$ $\left.\otimes\left|n_{\beta}\right\rangle,\left|\alpha^{-}\right\rangle\right\}$. This set of states will effectively represent the two lowest states of the signal mode in our detection scheme (photon absent or present).

The state $\left|\alpha^{-}\right\rangle$has energy $\omega_{\left|\alpha^{-}\right\rangle}=\omega_{\alpha}+g_{0, \alpha}+n_{\beta} \omega_{\beta}$. Resonantly irradiating with $\omega_{\left|\alpha^{-}\right\rangle}$will populate the corresponding state $\left|\alpha^{-}\right\rangle$only and, being an eigenstate, will not induce any internal dynamics.

Now we consider populating the $\beta$ mode with $n_{\beta}$ photons to dispersively detect the difference between the states $\mid$ vac, $\left.n_{\beta}\right\rangle \equiv\left|n_{\alpha}=0, \downarrow, n_{\beta}\right\rangle$ and $\left|\alpha^{-}\right\rangle$and thus the fact if a $\alpha$-mode photon has entered the cavity or not. So, we consider the coupling to the $\beta$ mode via the perturbation Hamiltonian

$$
\hat{H}_{\text {int }}=g_{0, \beta}\left(\hat{\sigma}^{+} \hat{a}_{\beta}+\hat{a}_{\beta}^{\dagger} \hat{\sigma}^{-}\right) .
$$

The idea is that the second-order energy shift of the transition frequency between states $\left|\mathrm{vac}, n_{\beta}\right\rangle$ and $\left|\alpha^{-}\right\rangle$will contain a term $\propto n_{\beta}$ which can be used to read off the effective coupling $g$ between the hybrid system of $\alpha$ mode (qubit) and the $\beta$ mode. We find that the system can be described by the effective Hamiltonian analogous to Eq. (1),

$$
H_{\text {eff }}=\hbar \omega_{s} \hat{a}_{s}^{\dagger} \hat{a}_{s}+\hbar \omega_{\beta} \hat{a}_{\beta}^{\dagger} \hat{a}_{\beta}+g \hat{a}_{s}^{\dagger} \hat{a}_{s} \hat{a}_{\beta}^{\dagger} \hat{a}_{\beta},
$$

with a renormalized transition frequency of our hybrid qubit/ cavity system given by

$$
\omega_{s} \equiv \omega_{\alpha}+g_{0, \alpha}-\frac{\left|g_{0, \beta}\right|^{2}}{2\left(\omega_{\beta}-\omega_{\alpha}\right)}
$$

and an effective cross-Kerr coupling of

$$
g \equiv-\frac{\left|g_{0, \beta}\right|^{2}}{\left(\omega_{\beta}-\omega_{\alpha}\right)}\left[1+\frac{g_{0, \alpha}}{\left(\omega_{\beta}-\omega_{\alpha}\right)}\right] \approx-\frac{\left|g_{0, \beta}\right|^{2}}{\left(\omega_{\beta}-\omega_{\alpha}\right)} .
$$

Note that in the newly introduced system with subscript $s$ (our hybrid system) only one excitation is possible such that $\left|n_{s}=1\right\rangle \equiv\left|\alpha^{-}\right\rangle$. 
In order to evaluate the result numerically for different modes, it is appropriate to use Eq. (5) and the following relations for the dependence of the parameters on the mode index:

$$
\begin{aligned}
g_{0, j} & =\sqrt{j} g_{0}, \\
\omega_{j} & =j \omega_{0}, \\
\kappa_{j} & =j \kappa_{0},
\end{aligned}
$$

which lead to the following expression for the coupling and for the dimensionless measurement rate:

$$
\begin{gathered}
g=-\frac{\left|g_{0}\right|^{2}}{\omega_{0}} \frac{\beta}{\beta-\alpha}, \\
\frac{\Gamma}{\kappa_{\alpha}}=\frac{|g|^{2}\left\langle\hat{n}_{\beta}\right\rangle}{4 \kappa_{\alpha} \kappa_{\beta}}=\left\langle\hat{n}_{\beta}\right\rangle \frac{\left|g_{0}\right|^{4}}{4 \omega_{0}^{2} \kappa_{0}^{2}} \frac{1}{\alpha \beta}\left(\frac{\beta}{\beta-\alpha}\right)^{2} .
\end{gathered}
$$

Assuming $\quad g_{0}=2 \pi \times 50 \mathrm{MHz}, \quad \kappa_{0}=10 \mathrm{MHz}, \quad \omega_{0}=2 \pi$ $\times 6 \mathrm{GHz}$, and $(\alpha, \beta)=(3,5)$ we observe that the optimal measurement rate of $\Gamma / \kappa=1 / 4$ is reached at a measurement mode photon number of $\left\langle\hat{n}_{\beta}\right\rangle \approx 35$, well below the critical photon number, where the dispersive approximation starts to break down as discussed, e.g., in [38]. Note that the dropped term in Eq. (14) amounts to a correction of only about $2 \%$ in the needed photon number.

Simply adjusting the decay rate of the cavity or the qubit resonator coupling will enable the experimentalist to observe all the features discussed in this paper as the full range of measurement rates are available, starting from the ideal detection limit $\Gamma / \kappa=1 / 4$ to the quantum Zeno limit $\Gamma / \kappa \gg 1$. The detector efficiency, although limited by the quantum Zeno effect as shown before, can then reach values of about $30 \%$ even without considering more elaborate detector and signal analysis schemes.

We note that in an alternative scheme, both the signal and the detection modes can be dispersively coupled to the qubit, although generically this would lead to a weaker overall coupling $g$. Finally, we remark that having control over the individual mode frequencies involved (e.g., in a setup with two cavities instead of one) would be advantageous for two reasons: first, it could avoid spurious higher-order processes in which the signal mode is contaminated by the decay of photons from the detection mode by making those processes strongly off resonant; second, it would allow to tune the signal and detection modes relatively close to each other, thereby enhancing the coupling.

\section{Possible realization using self-assembled quantum dots and photonic crystals}

Photonic crystal cavities have been proven to couple to excitons in self-assembled quantum dots inside the cavity. The system is in principle analogous to the circuit QED system discussed above (in the sense that the dot coupling to the cavity is well described by a Jaynes-Cummings Hamiltonian), however, the crucial difference is that photonic crystals and excitons operate at frequencies corresponding to vis- ible light. This makes measurement much harder due to the extremely short time spans involved compared to microwaves. However, if we assume the same coupling paradigm as before, we have to plug in the numbers typically found in present-day experiments [39-42]. Cavity resonance frequencies are, e.g., $\omega_{0}=2 \pi \times 0.3 \times 10^{6} \mathrm{GHz}$, the fundamental Jaynes-Cummings coupling of an exciton to the cavity mode is $g_{0} \approx 2 \pi \times 25 \mathrm{GHz}$, while present-day cavity decay rates also range in the $O(2 \pi \times 30 \mathrm{GHz})$ region. If the cavity quality factors could be improved by about a factor of 100 and the coupling of the cavity to the pump could be improved by integrating the pump source on the chip and connecting it with a waveguide to the cavity (the technology for both exists), then we can reach optimal detection efficiencies $\Gamma / \kappa$ $\approx 1 / 4$ with $O\left(10^{4}\right)$ photons in the detection mode. We therefore conclude that our scheme would also be applicable in the field of photonic crystals and self-assembled quantum dots once certain design improvements have been realized in those systems. Another experiment in which essentially the same physics could be observed is the detection of single photons in a microwave cavity by employing the dispersive interaction with a stream of Rydberg atoms [8].

\section{CONCLUSIONS}

In this paper we have analyzed a rather generic scheme for the detection of itinerant photons in a QND measurement process, employing quantum trajectory simulations. One particularly important and feasible implementation would be in superconducting circuit QED, where this scheme would enable the on-chip detection of single microwave photons. We have shown how the quantum Zeno effect enters the detection efficiency, a result that will be relevant to many other situations, such as the detection of electrons tunneling through a quantum dot by current passing through a nearby quantum point contact [43], the detection of itinerant phonons entering a micromechanical cantilever or membrane (e.g., in an optomechanical setup [44]), and other similar settings in mesoscopic physics, quantum optics, and atomic physics.

\section{ACKNOWLEDGMENTS}

We thank S. M. Girvin, J. M. Gambetta, A. A. Houck, M. Blencowe, A. Blais, J.-M. Raimond, and Dirk Englund for discussions. Support from the SFB 631, NIM, and the Emmy-Noether program (F.M.) of the DFG and EuroSQIP are gratefully acknowledged. E. S. thanks the Ikerbasque Foundation, the EU EuroSQIP project, and the UPV-EHU Grant GIU07/40.

\section{APPENDIX: DERIVATION OF THE ADIABATICALLY ELIMINATED STOCHASTIC MASTER EQUATION}

\section{Unraveling the master equation}

\section{a. Motivation}

The goal of this procedure is to obtain a stochastic master equation which models the system including the measurement back action and the corresponding noisy measurement 
signal in a consistent way starting from Eq. (2) which reads

$$
\begin{aligned}
\dot{\hat{\rho}}= & -i\left[\frac{\kappa_{1}}{2}\left(\alpha \hat{a}_{1}^{\dagger}+\alpha^{*} \hat{a}_{1}\right)+g \hat{a}_{1}^{\dagger} \hat{a}_{1} \hat{a}_{0}^{\dagger} \hat{a}_{0}, \hat{\rho}\right] \\
& +\kappa\left(\hat{a}_{0} \hat{\rho} \hat{a}_{0}^{\dagger}-\frac{1}{2} \hat{a}_{0}^{\dagger} \hat{a}_{0} \hat{\rho}-\frac{1}{2} \hat{\rho} \hat{a}_{0}^{\dagger} \hat{a}_{0}\right) \\
& +\kappa_{1}\left(\hat{a}_{1} \hat{\rho} \hat{a}_{1}^{\dagger}-\frac{1}{2} \hat{a}_{1}^{\dagger} \hat{a}_{1} \hat{\rho}-\frac{1}{2} \hat{\rho} \hat{a}_{1}^{\dagger} \hat{a}_{1}\right) \\
& -i \sqrt{\dot{N}_{\text {in }} / 2 \kappa}\left[\hat{a}_{0}+\hat{a}_{0}^{\dagger}, \hat{\rho}\right] .
\end{aligned}
$$

This way, we will gain insights beyond the ensemble-average description of the usual master equation which is crucial for the analysis the detection efficiency.

\section{b. Remark on stochastic master equations}

The master equation in general is an ensemble-average description of a quantum system. It can be thought of as arising from averaging a stochastic unraveled master equation.

Note that there is, without a physical concept of the processes involved, no unique way of obtaining a stochastic master equation from the averaged master equation $[33,34,45]$, a process which is known as unraveling. However, once we consider the physical details of the measurement, quantum mechanics allows us to unravel the master equation Eq. (A10) in such a way that we obtain (i) a classical measurement signal as produced by homodyne detection of the light field leaking out the detector mode and (ii) simultaneously a master equation conditioned on this measurement signal. This enables us to self-consistently simulate the measured signal and the quantum dynamics of our system which gave rise to exactly this signal.

\section{c. Outline of calculation}

We will observe that pure number states of the signal mode are attractor solutions. As a result of the measurement, the signal mode's state is stochastically forced toward a pure number state on a time scale that corresponds to the time that we need to extract the information about this number state from the measured signal. The attractor nature of the number states also becomes apparent from the fact that the stochastic term in the master equation becomes strictly zero as can be seen from Eq. (3).

In the following derivation, we follow Refs. [18,33-36]. Note that the choice of unraveling formally corresponds to projecting the field leaking out of the detector mode into the free space field modes (which we also call the measurement bath) onto the correct basis states. In this case we project onto the eigenstates of the field quadrature we are interested in to extract the phase shift imposed by a signal photon in the cavity. We start by writing the measurement bath as an infinite set of harmonic oscillators (e.g., the modes of a transmission line),

$$
\hat{H}_{\text {bath }}=\sum_{n} \omega_{d, n} \hat{b}_{d, n}^{\dagger} \hat{b}_{d, n},
$$

interacting with the detector mode by the Hamiltonian

$$
\hat{H}_{\mathrm{int}}=i \sum_{n} g_{d}\left(\omega_{n}\right)\left(\hat{b}_{d, n}^{\dagger} \hat{a}_{1}-\hat{b}_{d, n} \hat{a}_{1}^{\dagger}\right) .
$$

We employ the usual continuum and Markovian limit and define the detector mode decay rate $\kappa_{\mathrm{det}} \equiv \pi D\left(\omega_{1}\right)\left|g_{d}\left(\omega_{1}\right)\right|^{2}$, where $D$ is the density of states. [Note that this assumption cannot be easily relaxed for the purpose of deriving a stochastic master equation. It guarantees that the projective measurement of the bath will always disentangle system and bath which is not true for general non-Markovian baths]. Details have been recently discussed in [45]. The operator of the measured signal is then

$$
\begin{aligned}
\hat{X}(t)= & \sum_{n}\left[\hat{b}_{d, n}^{\dagger}(t)+\hat{b}_{d, n}(t)\right]=\sqrt{\kappa_{\operatorname{det}}}\left[\hat{a}_{1}(t)+\hat{a}_{1}^{\dagger}(t)\right] \\
& +\sum_{n}\left[\hat{b}_{d, n}^{\dagger}\left(t_{0}\right) e^{i \omega_{n}\left(t-t_{0}\right)}+\hat{b}_{d, n}\left(t_{0}\right) e^{-i \omega_{n}\left(t-t_{0}\right)}\right],
\end{aligned}
$$

where the last two lines represent the inhomogeneous and homogeneous parts of the solution of the equations of motion for the Heisenberg operators $\hat{b}_{d, n}$, respectively. Using $\left\langle\hat{b}_{n}\left(t_{0}\right)\right\rangle=\left\langle\hat{b}_{n}^{\dagger}\left(t_{0}\right)\right\rangle=0$, the average signal can be expressed as

$$
\langle\hat{X}(t)\rangle=\sqrt{\kappa_{\operatorname{det}}}\left\langle\hat{a}(t)+\hat{a}_{1}^{\dagger}(t)\right\rangle .
$$

Now the idea is to let this interaction Hamiltonian Eq. (A3) act for a small time $\Delta t$ before projecting the bath onto an eigenstate. Every time the bath is projected, the previously generated entanglement between bath and system ensures that this will also have a (slight) effect on the system state. By this mechanism it becomes clear that the random choice of an eigenvalue of the bath will in the continuum limit give rise to a stochastic term in the measured signal (noise) as well as in the system dynamics (back action). This random choice of eigenvalue/eigenstate of $\hat{X}$ happens according to the probability distribution given by

$$
P(X)=\langle X|\hat{\rho}(t+\Delta t)| X\rangle
$$

and projects the density matrix onto the eigenstate such that

$$
\hat{\rho} \mapsto \frac{|X\rangle\langle X|\hat{\rho}(t+\Delta t)| X\rangle\langle X|}{\langle X|\hat{\rho}(t+\Delta t)| X\rangle} .
$$

In order to simplify the notation, we introduce global bath

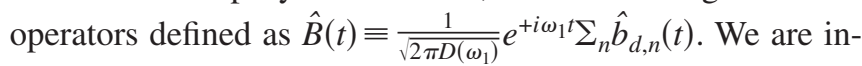
terested in the evolution of the operator $\hat{B}(t)$ over the time scale $\Delta t$ which is much shorter than the internal time scale of the intrinsic dynamics of $\hat{B}(t)$. Thus we can define an operator $d \hat{B}(t)$ as

$$
d \hat{B}(t) \equiv \frac{1}{\sqrt{\Delta t}} \int_{t}^{t+\Delta t} \hat{B}(\tau) d \tau
$$

To leading order in $\Delta t$, the evolution of the density matrix will then have the following form: 


$$
\begin{aligned}
\hat{\rho}(t+\Delta t)= & \hat{\rho}(t) \otimes \rho_{\text {bath }}(t)+\sqrt{\kappa_{\operatorname{det}} \Delta t}\left[d \hat{B} \hat{a}_{1}^{\dagger}+\hat{a}_{1} d \hat{B}^{\dagger}, \hat{\rho}(t)\right. \\
& \left.\otimes \hat{\rho}_{\text {bath }}(t)\right]+O(\Delta t) .
\end{aligned}
$$

Note that we have already worked out all $O(\Delta t)$ terms which constitute the deterministic evolution so we can focus on the $O(\sqrt{\Delta t})$ terms. If one does so and keeps all the terms up to order $O(\sqrt{\Delta t})$, one realizes that the state after measurement and projection will have the form

$$
\begin{aligned}
\Delta \hat{\rho}(t+\Delta t) & =\frac{|X\rangle\langle X|\hat{\rho}(t+\Delta t)| X\rangle\langle X|}{\langle X|\hat{\rho}(t+\Delta t)| X\rangle}-\hat{\rho}(t) \\
& =\sqrt{\Delta t} X\left[\hat{a}_{1} \hat{\rho}(t)+\hat{\rho}(t) \hat{a}_{1}^{\dagger}-\left\langle\hat{a}_{1}+\hat{a}_{1}^{\dagger}\right\rangle(t) \hat{\rho}(t)\right]+O(\Delta t) .
\end{aligned}
$$

Similarly we find for the signal $X$, assuming zero temperature, that the bath state at time $t$ is a Gaussian centered at $X=0$ and with width 1 . At time $t+\Delta t$, after interaction and projection, the bath will still be in a Gaussian state, but centered around $\sqrt{\kappa_{\operatorname{det}}}\left\langle\hat{a}_{1}+\hat{a}_{1}^{\dagger}\right\rangle \Delta t$. Thus we can identify the functional form of the noisy signal. It reads in differential form

$$
X(t)=\sqrt{\kappa_{\operatorname{det}}}\left(a_{1}+a_{1}^{\dagger}\right\rangle(t)+\xi(t),
$$

where $\xi(t)$ is white noise, with $\langle\xi(t)\rangle=0$ and $\left\langle\xi(t) \xi\left(t^{\prime}\right)\right\rangle$ $=\delta\left(t-t^{\prime}\right)$. Writing Eq. (A5) in differential form and inserting Eq. (A6) and again keeping terms up to $O(\Delta t)$, we find the stochastic contribution $\dot{\hat{\rho}}_{\text {st }}(t)$ of the master equation that describes the measurement back action given we have measured a certain signal trajectory $X(t)$ as

$$
\dot{\hat{\rho}}_{\mathrm{st}}(t)=\sqrt{\kappa_{\mathrm{det}}}\left[\hat{a}_{1} \hat{\rho}(t)+\hat{\rho}(t) \hat{a}_{1}^{\dagger}-\left\langle\hat{a}_{1}+\hat{a}_{1}^{\dagger}\right\rangle(t) \hat{\rho}(t)\right] \xi(t) .
$$

Together with the deterministic evolution of Eq. (A1), the unraveled master equation reads

$$
\begin{aligned}
\dot{\hat{\rho}}= & -i g\left[\hat{a}_{1}^{\dagger} \hat{a}_{1} \hat{a}_{0}^{\dagger} \hat{a}_{0}, \hat{\rho}\right]-i g\left[\left(\alpha^{*} \hat{a}_{1}^{\dagger}+\alpha \hat{a}_{1}\right) \hat{a}_{0}^{\dagger} \hat{a}_{0}, \hat{\rho}\right] \\
& +\kappa\left(\hat{a}_{0} \hat{\rho} \hat{a}_{0}^{\dagger}-\frac{1}{2} \hat{a}_{0}^{\dagger} \hat{a}_{0} \hat{\rho}-\frac{1}{2} \hat{\rho} \hat{a}_{0}^{\dagger} \hat{a}_{0}\right) \\
& \left.+\kappa_{1}\left(\hat{a}_{1} \hat{\rho} \hat{a}_{1}^{\dagger}-\frac{1}{2} \hat{a}_{1}^{\dagger} \hat{a}_{1} \hat{\rho}-\frac{1}{2} \hat{\rho} \hat{a}_{1}^{\dagger} \hat{a}_{1}\right)-i \sqrt{\dot{N}_{\text {in }} / 2 \kappa\left[\hat{a}_{0}\right.}+\hat{a}_{0}^{\dagger}, \hat{\rho}\right] \\
& +\sqrt{\kappa}\left[\hat{a}_{1} \hat{\rho}(t)+\hat{\rho}(t) \hat{a}_{1}^{\dagger}-\left\langle\hat{a}_{1}+\hat{a}_{1}^{\dagger}\right\rangle(t) \hat{\rho}(t)\right] \xi(t) .
\end{aligned}
$$

The last steps of this procedure can be found in more detail in [33]. Note that the stochastic term is not of Lindblad form but has nonlinear superoperator form [33].

\section{Adiabatic elimination of the detector mode}

The key assumption for the adiabatic elimination procedure to be correct and appropriate is that the dynamics of the ancilla system, in our case the detector mode, is much faster than the dynamics of the system, in our case the signal mode. In this limit $\kappa_{\mathrm{det}} / \kappa \gg 1$, the detector mode will relax to its displaced driven state on a time scale that is much faster than the intrinsic time scales in the signal mode.

The first step to be taken is to transform the master equation such that the phase-space origin for the detector mode is centered at its steady state under the coherent drive. This can be done by applying the displacement operator $\hat{D}(-\alpha)$ $=\exp \left[-\alpha \hat{a}_{1}^{\dagger}+\alpha^{\star} a_{1}\right]$ to Eq. (A1). As a result, we have eliminated the driving term for the detector mode and obtain as the new master equation

$$
\begin{aligned}
\dot{\hat{\rho}}= & -i g\left[\hat{a}_{1}^{\dagger} \hat{a}_{1} \hat{a}_{0}^{\dagger} \hat{a}_{0}, \hat{\rho}\right]-i g\left[\left(\alpha \hat{a}_{1}^{\dagger}+\alpha^{*} \hat{a}_{1}\right) \hat{a}_{0}^{\dagger} \hat{a}_{0}, \hat{\rho}\right] \\
& +\kappa\left(\hat{a}_{0} \hat{\rho} \hat{a}_{0}^{\dagger}-\frac{1}{2} \hat{a}_{0}^{\dagger} \hat{a}_{0} \hat{\rho}-\frac{1}{2} \hat{\rho} \hat{a}_{0}^{\dagger} \hat{a}_{0}\right) \\
& +\kappa_{1}\left(\hat{a}_{1} \hat{\rho} \hat{a}_{1}^{\dagger}-\frac{1}{2} \hat{a}_{1}^{\dagger} \hat{a}_{1} \hat{\rho}-\frac{1}{2} \hat{\rho} \hat{a}_{1}^{\dagger} \hat{a}_{1}\right) \\
& -i \sqrt{\dot{N}_{\text {in }} / 2 \kappa}\left[\hat{a}_{0}+\hat{a}_{0}^{\dagger}, \hat{\rho}\right] .
\end{aligned}
$$

Note that the new vacuum state that the detector mode relaxes to is actually the coherent state $|\alpha\rangle$. The first two terms capture the fluctuations in the ancilla excitation while we have eliminated the frequency renormalization term $-i|\alpha|^{2} g\left[\hat{a}_{0}^{\dagger} \hat{a}_{0}, \hat{\rho}\right]$ by absorbing it into the interaction picture.

The adiabatic elimination [18,33-35] is a well-controlled approximation and can be expressed as an expansion of the density matrix in a small parameter. Introducing this small parameter $\epsilon \ll 1$ such that $\kappa / \kappa_{\mathrm{det}}=O(\epsilon)$ and $g|\alpha| / \kappa_{\mathrm{det}}=O(\epsilon)$ are satisfied, we can formally expand the density matrix in orders of $\epsilon$ as follows:

$$
\begin{aligned}
\hat{\rho}= & \hat{\rho}_{s}^{(00)} \otimes \hat{\sigma}+\hat{\rho}_{s}^{(10)} \otimes \hat{a}_{1}^{\dagger} \hat{\sigma}+\hat{\rho}_{s}^{(01)} \otimes \hat{\sigma} \hat{a}_{1}+\hat{\rho}_{s}^{(11)} \otimes \hat{a}_{1}^{\dagger} \hat{\sigma} \hat{a}_{1} \\
& +\hat{\rho}_{s}^{(20)} \otimes \hat{a}_{1}^{\dagger 2} \hat{\sigma}+\hat{\rho}_{s}^{(02)} \otimes \hat{\sigma} \hat{a}_{1}^{2}+O\left(\epsilon^{3}\right) .
\end{aligned}
$$

Up to now, we have just rewritten the density matrix in a form that makes the different orders of $\epsilon$ as well as offdiagonal terms and diagonal terms apparent. $\hat{\sigma} \equiv|\mathrm{vac}\rangle\langle\mathrm{vac}|$ denotes the displaced coherent state of the detector mode which is a vacuum state. Using this decomposition, one can straightforwardly realize that taking the trace over the detector mode results in the following form for the signal mode density matrix:

$$
\hat{\rho}_{s}=\hat{\rho}_{s}^{(00)}+\hat{\rho}_{s}^{(11)} .
$$

We now evaluate Eq. (A8) term by term using Eq. (A9), $\left[\hat{a}_{1}, \hat{a}_{1}^{\dagger}\right]=1$, and the fact that the coherent state $\hat{\sigma}$ is a vacuum state. After lengthy but simple manipulation of the expressions we can eliminate all detector mode operators from the master equation. Key steps in this calculation are (a) replacing the off-diagonal terms in the signal mode density matrix by their steady-state solution, i.e., setting $\dot{\hat{\rho}}_{s}^{(10)}=\dot{\hat{\rho}}_{s}^{(01)}=\dot{\hat{\rho}}_{s}^{(02)}$ $=\dot{\hat{\rho}}_{s}^{(20)}=0$ and (b) disregarding off-diagonal terms in the detector mode density matrix such as, e.g., $\hat{a}_{1}^{\dagger} \hat{a}_{1}^{\dagger} \hat{a}_{1}^{\dagger} \operatorname{coh} \hat{\rho}_{\text {det }}$, which are further away from the diagonal than the expansion range of the ancilla state. The result (see [18]) reads

$$
\begin{aligned}
\dot{\hat{\rho}}= & -i \sqrt{\frac{\dot{N}_{\mathrm{in}} \kappa}{2}}\left[\hat{a}+\hat{a}^{\dagger}, \hat{\rho}\right]+\kappa\left(\hat{a} \hat{\rho} \hat{a^{\dagger}}-\frac{1}{2} \hat{n} \hat{\rho}-\frac{1}{2} \hat{\rho} \hat{n}\right) \\
& -2 \frac{g^{2}|\alpha|^{2}}{\kappa_{\operatorname{det}}}[\hat{n},[\hat{n}, \hat{\rho}]] .
\end{aligned}
$$

From now on we will introduce the measurement rate $\Gamma$ $\equiv g^{2}|\alpha|^{2} / \kappa_{\text {det }}$ which is the coefficient of the measurement 
induced diffusion term. This simplified master equation [Eq. (A10)] for the signal mode alone later enables us to perform detailed numerical studies due to the more manageable size of the density matrix. Furthermore, only in the adiabatically eliminated version of the master equation we will be able to explicitly see that the chosen limit of $\kappa_{\mathrm{det}} \gg \kappa$ exactly corresponds to measuring the photon number of the signal mode by looking at the phase shift on the detection mode.

The last step of the calculation follows the lines of the elimination of the detector mode operators from the deterministic terms in the master equation. However, to adiabatically eliminate the detector mode operators from the stochastic term, one more consideration is necessary. Simply replacing the off-diagonal terms by their stationary solution will not be enough as the stochastic term averages to zero. Instead we have to compute the variance of the stochastic term and integrate it over the time scale of the diagonal terms $\Delta t=\kappa_{\operatorname{det}}^{-1}$. Doing so, we find that the resulting master equation Eq. (3) reads

$$
\begin{aligned}
\hat{\rho}_{s}= & -i \sqrt{\frac{\dot{N}_{\text {in }} \kappa}{2}}\left[\hat{a}+\hat{a}^{\dagger}, \hat{\rho}\right]+\kappa\left(\hat{a} \hat{\rho} \hat{a}^{\dagger}-\frac{1}{2} \hat{n} \hat{\rho}-\frac{1}{2} \hat{\rho} \hat{n}\right) \\
& -2 \Gamma[\hat{n},[\hat{n}, \hat{\rho}]]-\sqrt{4 \Gamma}[\hat{n} \hat{\rho}+\hat{\rho} \hat{n}-2 \hat{\rho}\langle\hat{n}\rangle(t)] \xi(t) .
\end{aligned}
$$

For the rest of the paper (in the main text) we will drop the subscript $s$ which denotes the signal mode. In the course of the adiabatic elimination calculation we also find the very useful identity

$$
\sqrt{\kappa_{\mathrm{det}}}\left\langle\hat{a}_{1}+\hat{a}_{1}^{\dagger}\right\rangle(t)=2 \sqrt{2 \Gamma}\left\langle\hat{a}_{0}^{\dagger} \hat{a}_{0}\right\rangle(t),
$$

which allows us to write the measurement signal (rescaled) Eq. (4) as

$$
X(t) \equiv\langle\hat{n}\rangle(t)+\frac{1}{4} \sqrt{\frac{1}{\Gamma}} \xi(t) .
$$

Having obtained the final form of the adiabatically eliminated stochastic master equation, we can turn to the detector logic and the evaluation of the photon detection efficiency. This will be done by numerically integrating Eqs. (3) and (A13) and applying a suitable nonlinear filter to the signal followed by a statistical analysis of the dependence of the photon count rate on the physical parameters in the system, especially $\Gamma / \kappa$, as explained in the main text.
[1] V. B. Braginsky, Y. I. Vorontsov, and K. S. Thorne, Science 209, 547 (1980).

[2] V. B. Braginsky and F. Y. Khalili, Quantum Measurement (Cambridge University Press, Cambridge, England, 1992).

[3] A. Wallraff, D. I. Schuster, A. Blais, L. Frunzio, J. Majer, M. H. Devoret, S. M. Girvin, and R. J. Schoelkopf, Phys. Rev. Lett. 95, 060501 (2005).

[4] D. I. Schuster, A. A. Houck, J. A. Schreier, A. Wallraff, J. M. Gambetta, A. Blais, L. Frunzio, J. Majer, B. Johnson, M. H. Devoret, S. M. Girvin, and R. J. Schoelkopf, Nature (London) 445, 515 (2007).

[5] J. Gambetta, W. A. Braff, A. Wallraff, S. M. Girvin, and R. J. Schoelkopf, Phys. Rev. A 76, 012325 (2007).

[6] J. Gambetta, A. Blais, M. Boissonneault, A. A. Houck, D. I. Schuster, and S. M. Girvin, Phys. Rev. A 77, 012112 (2008).

[7] J. I. Cirac, R. Blatt, A. S. Parkins, and P. Zoller, Phys. Rev. Lett. 70, 762 (1993).

[8] S. Gleyzes, S. Kuhr, C. Guerlin, J. Bernu, S. Deléglise, U. Busk Hoff, M. Brune, J.-M. Raimond, and S. Haroche, Nature (London) 446, 297 (2007).

[9] W. Nagourney, J. Sandberg, and H. Dehmelt, Phys. Rev. Lett. 56, 2797 (1986).

[10] M. Porrati and S. Putterman, Phys. Rev. A 36, 929 (1987).

[11] S. Peil and G. Gabrielse, Phys. Rev. Lett. 83, 1287 (1999).

[12] B. Misra and E. C. G. Sudarshan, J. Math. Phys. 18, 756 (1977).

[13] W. M. Itano, D. J. Heinzen, J. J. Bollinger, and D. J. Wineland,
Phys. Rev. A 41, 2295 (1990).

[14] K. Jacobs, P. Lougovski, and M. Blencowe, Phys. Rev. Lett. 98, 147201 (2007).

[15] R. Rossi, Jr., M. Nemes, and A. de Magalhaes, Phys. Rev. A. 77, 012107 (2008).

[16] J. A. Damborenea, I. L. Egusquiza, G. C. Hegerfeldt, and J. G. Muga, Phys. Rev. A 66, 052104 (2002).

[17] X.-B. Wang, J. Q. You, and F. Nori, Phys. Rev. A 77, 062339 (2008).

[18] D. H. Santamore, A. C. Doherty, and M. C. Cross, Phys. Rev. B 70, 144301 (2004).

[19] K. Jacobs, A. Jordan, and E. Irish, EPL 82, 18003 (2008).

[20] A. Wallraff, D. I. Schuster, A. Blais, L. Frunzio, R.-S. Huang, J. Majer, S. Kumar, S. M. Girvin, and R. J. Schoelkopf, Nature (London) 431, 162 (2004).

[21] A. A. Houck, D. I. Schuster, J. M. Gambetta, J. A. Schreier, B. R. Johnson, J. M. Chow, L. Frunzio, J. Majer, M. H. Devoret, S. M. Girvin and R. J. Schoelkopf, Nature (London) 449, 328 (2007).

[22] J. Johansson, S. Saito, T. Meno, H. Nakano, M. Ueda, K. Semba, and H. Takayanagi, Phys. Rev. Lett. 96, 127006 (2006).

[23] F. Helmer, M. Mariantoni, A. G. Fowler, J. von Delft, E. Solano, and F. Marquardt, EPL 85, 50007 (2009).

[24] M. Mariantoni, M. Storcz, F. Wilhelm, W. Oliver, A. Emmert, A. Marx, R. Gross, H. Christ, and E. Solano, e-print arXiv:cond-mat/0509737. 
[25] F. Marquardt, Phys. Rev. B 76, 205416 (2007).

[26] N. Imoto, H. A. Haus, and Y. Yamamoto, Phys. Rev. A 32, 2287 (1985).

[27] P. Yeh, J. Opt. Soc. Am. B 3, 747 (1986).

[28] H. A. Bachor, M. D. Levenson, D. F. Walls, S. H. Perlmutter, and R. M. Shelby, Phys. Rev. A 38, 180 (1988).

[29] M. Mariantoni, F. Deppe, A. Marx, R. Gross, F. K. Wilhelm, and E. Solano, Phys. Rev. B 78, 104508 (2008).

[30] A. Barchielli, Phys. Rev. A 34, 1642 (1986).

[31] H. Carmichael, An Open Systems Approach to Quantum Optics, Springer Lecture Notes Monographs Series No. 18 (Springer-Verlag, Berlin, 1993).

[32] D. F. Walls and G. J. Milburn, Quantum Optics (Springer, New York, 1995).

[33] H. M. Wiseman, Ph.D. thesis, University of Queensland, 1994.

[34] H. M. Wiseman and G. J. Milburn, Phys. Rev. A 49, 1350 (1994).

[35] A. C. Doherty and K. Jacobs, Phys. Rev. A 60, 2700 (1999).

[36] D. H. Santamore, Ph.D. thesis, California Institute of Technology, 2003.

[37] A. Blais, J. Gambetta, C. Cheung, R. Schoelkopf, and S. M. Girvin (unpublished).
[38] J. Gambetta, A. Blais, D. I. Schuster, A. Wallraff, L. Frunzio, J. Majer, M. H. Devoret, S. M. Girvin, and R. J. Schoelkopf, Phys. Rev. A 74, 042318 (2006); e-print arXiv:cond-mat/ 0602322.

[39] D. Englund, A. Majumdar, A. Faraon, M. Toishi, N. Stoltz, P. Petroff, and J. Vuckovic, e-print arXiv:0902.2428.

[40] D. Englund, I. Fushman, A. Faraon, and J. Vučković, Photonics Nanostruct. Fundam. Appl. 7, 56 (2009).

[41] M. Kaniber, A. Neumann, A. Laucht, M. F. Huck, M. Bichler, M.-C. Amann, and J. J. Finley, New J. Phys. 11, 013031 (2009).

[42] A. Laucht, F. Hofbauer, N. Hauke, J. Angele, S. Stobbe, M. Kaniber, G. Böhm, P. Lodahl, M. Amann, and J. Finley, New J. Phys. 11, 023034 (2009); e-print arXiv:0810.3010.

[43] E. Buks, R. Schuster, M. Heiblum, D. Mahalu, and V. Umansky, Nature (London) 391, 871 (1998).

[44] J. D. Thompson, B. M. Zwickl, A. M. Jayich, F. Marquadt, S. M. Girvin, and J. G. E. Harris, Nature (London) 452, 72 (2008).

[45] H. M. Wiseman and J. M. Gambetta, Phys. Rev. Lett. 101, 140401 (2008). 